\title{
On the $q$-analogue of the sum of cubes
}

\author{
S. Ole Warnaar* \\ Department of Mathematics and Statistics, \\ The University of Melbourne, VIC 3010, Australia \\ warnaar@ms . unimelb.edu . au
}

Submitted: Apr 7, 2004; Accepted: Aug 17, 2004; Published: August 23, 2004

2000 Mathematics Subject Classification: 05A19

\begin{abstract}
A simple $q$-analogue of the sum of cubes is given. This answers a question posed in this journal by Garrett and Hummel.
\end{abstract}

\section{The sum of cubes and its $q$-analogues}

It is well-known that the first $n$ consecutive cubes can be summed in closed form as

$$
\sum_{k=1}^{n} k^{3}=\left(\begin{array}{c}
n+1 \\
2
\end{array}\right)^{2}
$$

Recently, Garrett and Hummel discovered the following $q$-analogue of this result:

$$
\sum_{k=1}^{n} q^{k-1} \frac{\left(1-q^{k}\right)^{2}\left(2-q^{k-1}-q^{k+1}\right)}{(1-q)^{2}\left(1-q^{2}\right)}=\left[\begin{array}{c}
n+1 \\
2
\end{array}\right]^{2}
$$

where

$$
\left[\begin{array}{l}
n \\
k
\end{array}\right]=\frac{\left(1-q^{n-k+1}\right)\left(1-q^{n-k+2}\right) \cdots\left(1-q^{n}\right)}{(1-q)\left(1-q^{2}\right) \cdots\left(1-q^{k}\right)}
$$

is a $q$-binomial coefficient.

In their paper Garrett and Hummel commiserate the fact that (1) is not as simple as one might have hoped, and ask for a simpler sum of $q$-cubes. In response to this I propose the identity

$$
\sum_{k=1}^{n} q^{2 n-2 k} \frac{\left(1-q^{k}\right)^{2}\left(1-q^{2 k}\right)}{(1-q)^{2}\left(1-q^{2}\right)}=\left[\begin{array}{c}
n+1 \\
2
\end{array}\right]^{2} .
$$

${ }^{*}$ Work supported by the Australian Research Council 
Proof. Since

$$
\left[\begin{array}{c}
n+1 \\
2
\end{array}\right]^{2}-q^{2}\left[\begin{array}{l}
n \\
2
\end{array}\right]^{2}=\frac{\left(1-q^{n}\right)^{2}\left(1-q^{2 n}\right)}{(1-q)^{2}\left(1-q^{2}\right)}
$$

equation (2) immediately follows by induction on $n$.

The form of (2) should not really come as a surprise in view of the fact that the $q$-analogue of the sum of squares

$$
\sum_{k=1}^{n} k^{2}=\frac{1}{6} n(n+1)(2 n+1)
$$

is given by

$$
\sum_{k=1}^{n} q^{2 n-2 k} \frac{\left(1-q^{k}\right)\left(1-q^{3 k}\right)}{(1-q)\left(1-q^{3}\right)}=\frac{\left(1-q^{n}\right)\left(1-q^{n+1}\right)\left(1-q^{2 n+1}\right)}{(1-q)\left(1-q^{2}\right)\left(1-q^{3}\right)}
$$

and the $q$-analogue of

$$
\sum_{k=1}^{n} k=\left(\begin{array}{c}
n+1 \\
2
\end{array}\right)
$$

is

$$
\sum_{k=1}^{n} q^{2 n-2 k} \frac{\left(1-q^{k}\right)}{(1-q)}=\left[\begin{array}{c}
n+1 \\
2
\end{array}\right]
$$

\section{References}

[1] K. C. Garrett and K. Hummel, A combinatorial proof of the sum of q-cubes, Electron. J. Combin. 11 (2004), R9, 6pp. 